\title{
IL-33 restricts invasion and adhesion of trophoblast cell line JEG3 by downregulation of integrin $\alpha 4 \beta 1$ and CD62L
}

\author{
XIAO-HUI WANG ${ }^{1 *}$, WEI LIU ${ }^{1 *}$, DENG-XUAN FAN ${ }^{1}$, WEN-TING HU ${ }^{1}$, \\ MING-QING LI ${ }^{1}$, XIAO-YONG ZHU ${ }^{1}$ and LI-PING JIN ${ }^{1,2}$ \\ ${ }^{1}$ Laboratory for Reproductive Immunology, Hospital of Obstetrics and Gynecology, Fudan University \\ Shanghai Medical College, Shanghai 200011; ${ }^{2}$ Clinical and Translational Research Center, Shanghai First \\ Maternity and Infant Hospital, Tongji University School of Medicine, Shanghai 200040, P.R. China
}

Received December 23, 2015; Accepted January 27, 2017

DOI: $10.3892 / \mathrm{mmr} .2017 .7085$

\begin{abstract}
Interleukin-33 (IL-33) promotes migration of cancer cells through downregulating the expression of E-cadherin. Previous studies have demonstrated that IL-33 stimulates the proliferation of trophoblasts. However, the effect of IL-33 on the adhesion and invasion of trophoblasts has not been investigated in detail. In the present study, the expression of IL-33 and its receptor, IL-1 receptor-like 1 (ST2), was examined in villi from women during early pregnancy using immunohistochemistry. ST2 expression on human trophoblast and choriocarcinoma cell lines JAR, BeWo, JEG3 and HTR8 was confirmed by flow cytometry (FCM) assay. The effect of recombinant human IL-33 (rhIL-33) on adhesion, invasion and associated molecules was analyzed by cell adhesion, Matrigel invasion and FCM assays. The current study identified that human trophoblasts expressed IL-33 and ST2. RhIL-33 inhibited trophoblast invasion and adhesion, and decreased adhesion and invasion-associated molecules such as integrin $\alpha 4 \beta 1$ and CD62L. Therefore, these results suggest that IL-33 may serve an important role in limiting invasion and implantation of trophoblasts by adhesion and invasion-associated molecules, contributing to the formation of the placenta and maintenance of normal pregnancy during early pregnancy.
\end{abstract}

\section{Introduction}

Embryo implantation, decidualization and placentation are crucial steps in a successful establishment of mammalian

Correspondence to: Professor Li-Ping Jin, Laboratory for Reproductive Immunology, Hospital of Obstetrics and Gynecology, Fudan University Shanghai Medical College, 413 Zhao Zhou Road, Shanghai 200011, P.R. China

E-mail: jinlp01@163.com

*Contributed equally

Key words: interleukin-33, interleukin 1 receptor-like 1, trophoblasts, JEG3 cell line, invasion, adhesion pregnancy (1). Human embryo implantation involves a complicated network of molecular signaling modulated by endocrine and paracrine pathways (2). The trophoblast cell, a special type of epithelial cell, at the maternal-fetal interface is of high proliferation and invasion ability and is vital to embryo implantation and placenta formation $(3,4)$. During early human pregnancy, extravillous trophoblast cells (EVTs) from the placenta invade uterine decidual spiral arterioles and mediate the vascular remodeling (5). As a result, the placenta is supplied with more blood due to low blood pressure and high blood flow, all of which is essential to facilitate the normal growth and development of the fetus (6). Failure of invasion of the uterine wall may result in clinical pregnancy complications, including pre-eclampsia, fetal growth restriction and spontaneous abortion, while excess invasion may lead to gestational trophoblastic disease (7).

Embryo implantation and placentation are dynamic cellular events that require synchrony between the maternal environment and the embryo, in addition to complicated cell-cell communication between the implanting blastocyst and the receptive endometrium (8). A multitude of factors including endocrine regulators, oxygen concentration and immune cells contribute to the regulation of EVT function (8-10). Although EVTs possess a strong ability for invasion, they rarely exhibit infinite proliferation and distant metastasis. This indicates that adhesion and invasion of trophoblasts maintains dynamic balance due to regulation of cytokines directly or indirectly.

Interleukins are a series of bioactive molecules that regulate numerous important biological functions, including delivering messages between cells, activating the immune system, mediating the activation, proliferation and differentiation of $\mathrm{T} / \mathrm{B}$ cells, regulating non-specific immune response and mediating inflammation. Interleukin-33 (IL-33) is a novel member of the IL-1 superfamily of cytokines (expressed predominantly by stromal cells) $(11,12)$ and is associated with numerous diseases, including systemic sclerosis, inflammatory bowel disease, rheumatoid arthritis and systemic lupus erythematosus. Furthermore, IL-33 regulates the expression of adhesion molecules and promotes migration of cancer cells (13-20).

A previous study demonstrated that IL-33 and its receptor ST2 have been verified to be expressed in decidual tissue during the first trimester of pregnancy (21). In addition, IL-33 is 
reported to promote the proliferation and invasion of decidual stromal cells by upregulating CCL2/CCR2 via the signaling pathway of nuclear factor $\kappa \mathrm{B}$ and extracellular signal-related kinase (ERK) 1/2 (21). IL-33 secreted by macrophages at the maternal-fetal interface has been identified to activate trophoblast cells via protein kinase B (AKT) and ERK1/2 signaling pathways, promote the proliferation of trophoblast cells and serve an essential role in formation of placenta (22). However, the effect of IL-33 on the biological function of trophoblasts, such as adhesion and invasion, remains to be investigated. The aim of the present study was to investigate the effect of IL-33 on the adhesion and invasion of trophoblasts, and to explore the underlying mechanisms.

\section{Materials and methods}

Tissue collection. Human villous tissues were obtained from 10 healthy women during early pregnancy (age, $28.12 \pm 5.48$ years; gestational age at sampling, $52.37 \pm 5.01$ days, mean \pm standard deviation), whereby the pregnancy was terminated for non-medical reasons. All normal pregnancies were confirmed by ultrasound and blood test. Exclusion criteria included endocrine, anatomical and genetic reasons, infection and smoking. All procedures involving participants in the present study were approved by the Human Research Ethics Committee of Obstetrics and Gynecology Hospital, Fudan University (Shanghai, China), and all subjects provided informed consent to collect tissue samples.

Cell line and reagents. All cell lines are come from Laboratory for Reproductive Immunology, Hospital of Obstetrics and Gynecology, Fudan University Shanghai Medical College. The human trophoblast cell line HTR8/SVneo and the human choriocarcinoma cell line JEG3 were cultured in DMEM/F12 medium (Gibco; Thermo Fisher Scientific, Inc., Waltham, MA, USA), the human choriocarcinoma cell line JAR was cultured in RPMI-1640 medium (Gibco; Thermo Fisher Scientific, Inc.), and BeWo was cultured in Ham's F-12 medium (Gibco; Thermo Fisher Scientific, Inc.) supplemented with $10 \%$ fetal bovine serum (FBS; HyClone; GE Healthcare Life Sciences, Logan, UT, USA) in a humidified incubator at $37^{\circ} \mathrm{C}$ and $5 \%$ $\mathrm{CO}_{2}$.

Immunohistochemistry. The villous tissues were incubated $4 \%$ paraformaldehyde overnight at room temperature and embedded in paraffin. Paraffin-embedded villi specimens (thickness, $5 \mu \mathrm{m}$ ) were dewaxed in xylene, rehydrated in graded ethanol were incubated with hydrogen peroxide and $1 \%$ bovine serum albumin (BSA; 0332; Amresco, Solon, OH, USA)/TBS. Thereafter, the samples were incubated with rabbit anti-human IL-33 antibody (1 $\mu \mathrm{g} / \mathrm{ml}$; cat. no. HPA02442; Sigma-Aldrich; Merck Millipore, Darmstadt, Germany), rabbit anti-human ST2 antibody (10 $\mu \mathrm{g} / \mathrm{ml}$; cat. no. PRS3363; Sigma-Aldrich; Merck Millipore) or rabbit IgG isotype (1:2,000; cat. no. GTX35035; GeneTex, Inc., Irvine, CA, USA) overnight at $4^{\circ} \mathrm{C}$ in a humidified chamber. Following washing three times with TBS, the sections were overlaid with peroxidase-conjugated goat anti-rabbit IgG (Golden Bridge International, Inc., Bejing, China); the reaction was developed with DAB and counterstained with hematoxylin. The experiments were repeated ten times. All images were captured by Olympus D70 light microscopy (Olympus Corporation, Tokyo, Japan).

Flow cytometry (FCM) assay. HTR8/SVneo, JEG3, JAR and BeWo cells were treated with trypsin and transferred into plastic tubes after cultured at a density of $2 \times 10^{5}$ cells/well in 6 -well microplates for $48 \mathrm{~h}$. Cells were fixed in $4 \%$ paraformaldehyde for $20 \mathrm{~min}$ at room temperature, washed in PBS and permeabilized for $20 \mathrm{~min}$ in $0.1 \%$ Triton X-100-PBS. They were then washed and suspended in PBS, incubated with ST2-allophycocyanin-labeled antibodies (R\&D Systems, Inc., Minneapolis, MN, USA) for $30 \mathrm{~min}$ at room temperature. Next, cells were washed and suspended in PBS, and immediately analyzed by four-color FCM using CellQuest Pro software, version 5.1 (FACSCalibur; BD Biosciences, Franklin Lakes, NJ, USA) with an isotypic control (BioLegend, San Diego, CA, USA).

JEG3 cells were cultured at a density of $2 \times 10^{5}$ cells/well in 6-well microplates, and treated with recombinant human (rh)IL-33 (at 0 and $1 \mathrm{ng} / \mathrm{ml}$ ) for $48 \mathrm{~h}$, then the expression of integrin $\alpha 3 \beta 1$, integrin $\alpha 4 \beta 1$, integrin $\alpha 5 \beta 1$, integrin $\alpha 6 \beta 1$ and integrin $\alpha v \beta 3$ (R\&D Systems, Inc.), CD44, CD62L and E-cadherin (BioLegend, Inc., San Diego, CA, USA) on JEG3 cells was evaluated by FCM.

Cell adhesion assay. Cell adhesion to the extracellular matrix (ECM) was analyzed using a cell adhesion assay kit (CBA-070; CytoSelect 48-well Cell Adhesion Assay; Cell Biolabs, Inc., San Diego, CA, USA). JEG3 cells were cultured at a density of $2 \times 10^{5}$ cells/well in 6 -well microplates, treated with rhIL-33 (1 or $100 \mathrm{ng} / \mathrm{ml}$ ) for $48 \mathrm{~h}$, then treated with EDTA-free trypsin (SV30037.01; HyClone; GE Healthcare Life Sciences). Subsequently, a cell suspension in serum free media at the concentration of $5 \times 10^{5}$ cells $/ \mathrm{ml}$ was prepared. A total of $150 \mu 1$ of the cell suspension was added to the inside of each well (BSA-coated wells were provided as a negative control). The wells were incubated for $60 \mathrm{~min}$ in a cell culture incubator $\left(37^{\circ} \mathrm{C}, 5 \% \mathrm{CO}_{2}\right.$ atmosphere). The media was carefully discarded from each well and each well was gently washed 5 times with $250 \mu \mathrm{l}$ PBS. PBS was aspirated from each well and $200 \mu \mathrm{l}$ of Cell Stain Solution (included in cell adhesion assay kit) was added. This was incubated for $10 \mathrm{~min}$ at room temperature. The Cell Stain Solution was discarded from the wells and each well was gently washed 5 times with $500 \mu$ l deionized water. The final wash was discarded and the wells were left to air dry. A total of $200 \mu \mathrm{l}$ Extraction Solution/well was added, and then incubated $10 \mathrm{~min}$ on an orbital shaker. Each extracted sample had $150 \mu \mathrm{l}$ added to a 96-well microtiter plate and the OD $(570 \mathrm{~nm})$ was measured using a microplate reader.

Matrigel invasion assays. The invasion of JEG3 cells through Matrigel was evaluated objectively by counting the number of the cells that have transferred through the membrane in invasion chamber. The upper surface of the filter $(8 \mu \mathrm{m}$ pore size, $6.5 \mathrm{~mm}$ diameter, PET membrane; Corning Life Sciences, Corning, NY, USA) in Transwell plates (24-well plate, Corning, NY, USA) was pre-coated with $15 \mathrm{ml}$ Matrigel (BD Biosciences), and the filter was air-dried under sterile conditions. Prior to use, the Matrigel was rehydrated with $100 \mu 1$ warm DMEM/F12 medium (Gibco; Thermo Fisher 
Scientific, Inc.) for $2 \mathrm{~h}$. JEG3 cells $\left(1 \times 10^{5}\right)$ in $100 \mu \mathrm{l}$ serum free DMEM/F12 were seeded to the upper part of the chamber. A total volume of $600 \mu \mathrm{l}$ DMEM/F12 with $10 \%$ FBS was added to the lower chamber. RhIL-33 (at 1 or $100 \mathrm{ng} / \mathrm{ml}$ ) was added into lower chamber, and the cells were allowed to invade and held for $48 \mathrm{~h}$ in $5 \% \mathrm{CO}_{2}$ at $37^{\circ} \mathrm{C}$. Thereafter, the cells attached to the upper surface of the filter were removed by scrubbing with a cotton swab, and the cells remaining on the lower surface were fixed in methanol for $15 \mathrm{~min}$ at room temperature and stained with hematoxylin for $10 \mathrm{sec}$ at room temperature. For quantification, the cells that had migrated to the lower surface were counted under a light microscope in five random fields at a magnification of $x 100$ using the Olympus BX51 + DP70 microscopes with Adobe Photoshop CS4, version 11.0 (Adobe, San Jose, CA, USA). The results were expressed as a percentage of the controls (RhIL-33 at $0 \mathrm{ng} / \mathrm{ml}$ ). Each experiment was carried out in triplicate on three replicates.

Statistical analysis. All values are presented as mean \pm standard. Statistical comparisons were evaluated by unpaired two-sided t-tests or one-way analysis of variance using GraphPad Prism software (version 5.0; GraphPad Software, Inc., La Jolla, CA, USA). The post-hoc Dunnett test was used to compare significance levels between controls and various treatments. $\mathrm{P}<0.05$ was considered to indicate a statistically significant difference.

\section{Results}

$I L-33 / S T 2$ is co-expressed in trophoblasts. To explore the expression of IL-33 and its receptor ST2 at the maternal-fetal interface, immunohistochemistry was performed with paraffin-embedded human first-trimester villi tissues. As presented in Fig. 1, IL-33 and ST2 were co-expressed in trophoblasts of villi tissues. IL-33 was primarily expressed in the cytoplasm of trophoblast cells, whereas ST2 localized to the cell cytoplasm and membrane of trophoblast cells. These results indicated that IL-33/ST2 likely serves an important role in the formation and function of placenta, and IL-33 may regulate the biological function of trophoblast cells in an autocrine-dependent manner.

Trophoblast cell lines express ST2. The expression of ST2 in cell lines of JEG3, JAR, BeWo and HTR8 cells was detected by FCM assay. As demonstrated in Fig. 2, the highest expression of ST2 was observed in the BeWo cell line, with the lowest detected in HTR8 cell $(\mathrm{P}<0.01$ or $\mathrm{P}<0.001)$. According to a previous study, JEG3 spheroids presented the highest incidence of attachment and the greatest amount of invasion into the underlying endometrial stroma. Therefore, the JEG3 cell line was selected in subsequent trials.

IL-33 limits the adhesion to ECM and invasion of JEG3 cells. To investigate the effect of IL-33 on the adhesion and invasion of trophoblast cells, a matrix adhesion assay was performed to test the adhesion of JEG3 cell following treatment with rhIL-33 for 48 h. As indicated in Fig. 3, 1 and $100 \mathrm{ng} / \mathrm{ml}$ rhIL-33 stimulation led to a significant reduction in adhesion ability of JEG3 cells to fibronectin, laminin I, collagen IV and
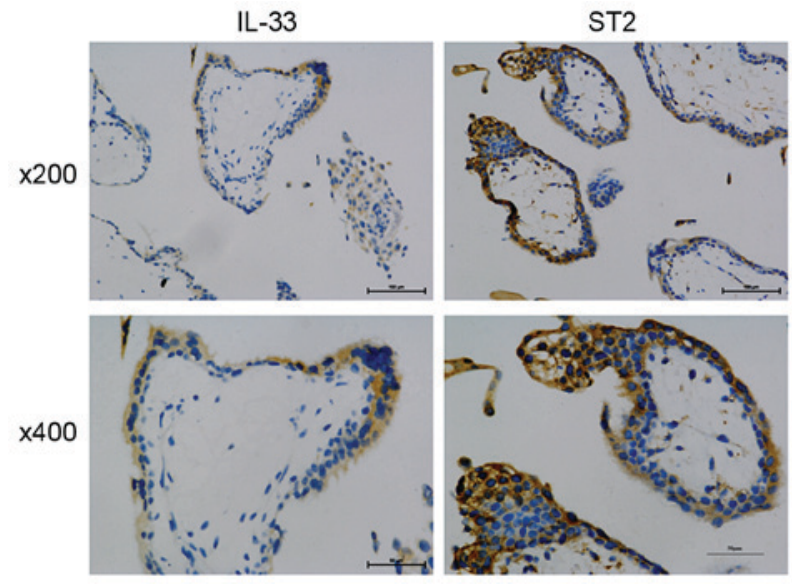

Figure 1. IL-33/ST2 is co-expressed in trophoblasts. Immunohistochemical analysis $(n=10)$ for IL-33 and ST2 expression in villi tissues from healthy women at the first-trimester of a normal pregnancy. Magnification, x200 or x400. IL-33, interleukin 33; ST2, IL-1 receptor-like 1.

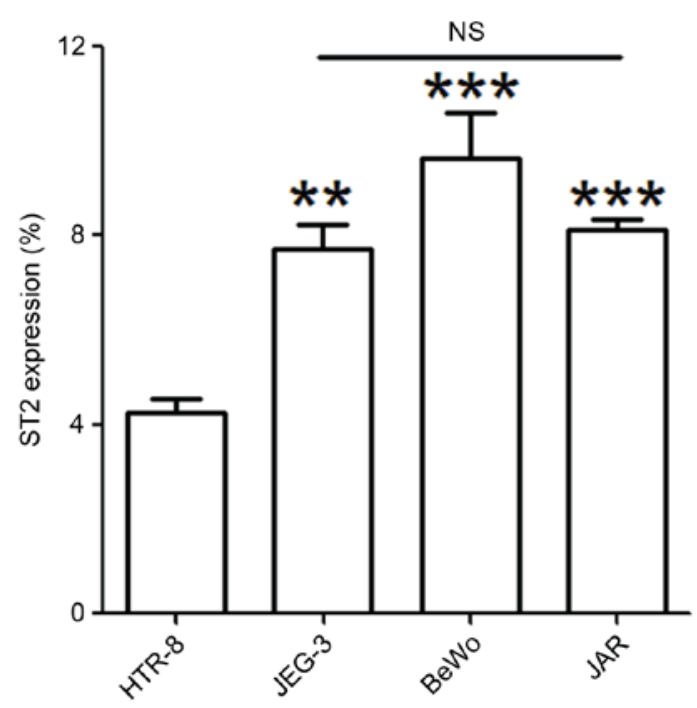

Figure 2. Trophoblast cell lines express ST2. Expression of ST2 in JEG3, JAR, BeWo and HTR8 cells was determined by flow cytometry. The data are expressed as the mean \pm standard error of the mean. ${ }^{* *} \mathrm{P}<0.01$ and ${ }^{* * *} \mathrm{P}<0.01$ vs. HTR8 group. ST2, interleukin 1 receptor-like 1; NS, no statistical significance.

fibrinogen (Fig. 3; $\mathrm{P}<0.01$ or $\mathrm{P}<0.001$ ). However, treatment with IL-33 had no influence on adhesion to collagen I (Fig. 3; $\mathrm{P}>0.05$ ).

In addition, the invasiveness of JEG3 cells with or without rhIL-33 was evaluated by a Matrigel invasion assay. rhIL-33 significantly restricted JEG3 cell invasion, especially at the concentration with $100 \mathrm{ng} / \mathrm{ml}$ (Fig. 4; P<0.001). These results suggested that IL-33 regulated trophoblast implantation at the maternal-fetal interface by limiting adhesion and invasion, thus maybe involved in regulating the formation of placenta and maintenance of normal pregnancy.

$I L-33$ reduces the expression of integrin $\alpha 4 \beta 1$ and CD62L on $J E G 3$ cells. To further explore the molecular mechanism of IL-33 on trophoblast adhesion and invasion, the expression of adhesion and invasion-associated molecules was evaluated in 


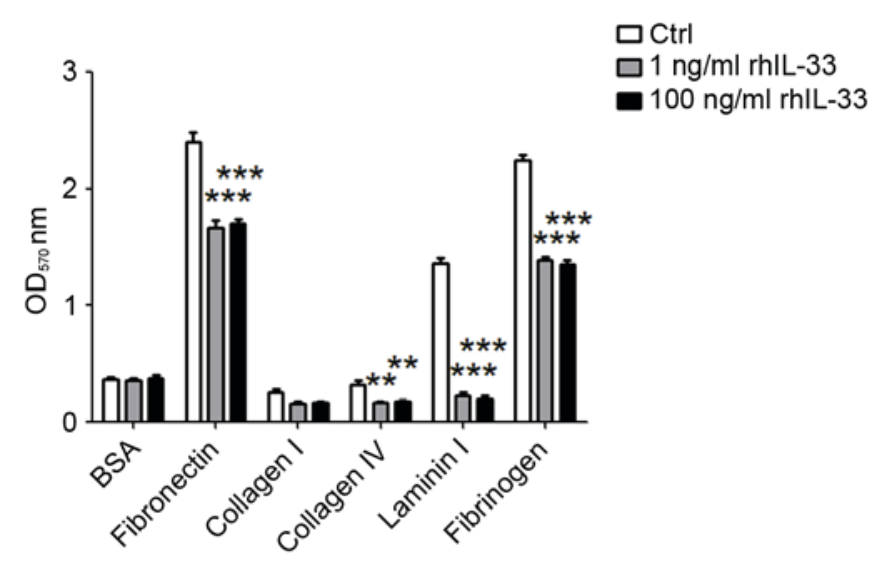

Figure 3. IL-33 limits the adhesion of JEG3 cells to extracellular matrix components. Following stimulation with 1 or $100 \mathrm{ng} / \mathrm{ml}$ rhIL-33 for $48 \mathrm{~h}$, the adhesion of JEG3 cells to fibronectin, laminin I, collagen I, collagen IV and fibrinogen were analyzed by cell adhesion assay, with BSA as the control. The data are expressed as the mean \pm standard error. ${ }^{* *} \mathrm{P}<0.01$ and ${ }^{* * * *} \mathrm{P}<0.01$ vs. control group. IL-33, interleukin 33; Ctrl, control; rhIL-33, recombinant human IL-33; BSA, bovine serum albumin.

the JEG3 cell line stimulated with or without rhIL-33 by FCM, including integrins, E-cadherin, CD62L and CD44. Almost $100 \%$ of JEG3 cells expressed integrin $\alpha 3 \beta 1$, integrin $\alpha 5 \beta 1$, integrin $\alpha 6 \beta 1$ and E-cadherin (Fig. 5). CD44 and integrin $\alpha v \beta 3$ were nearly unexpressed in JEG3 cells. Among these, the expression of integrin $\alpha 4 \beta 1$ and CD62L became significantly inhibited by rhIL-33, implying that IL-33 might decrease the adhesion and invasion of trophoblasts through downregulating integrin $\alpha 4 \beta 1$ and CD62L.

Conclusively, IL-33 and its receptor ST2 are expressed in trophoblast cells, which may participate in regulating the biological function of trophoblast cells. The results, using the JEG3 cell line (a trophoblast cell line with high adhesion and invasion ability), indicates the presence of rhIL-33 results in a decrease of trophoblast adhesion and invasion. The possible underlying mechanism is that IL-33 reduces the expression of adhesion and invasion-associated molecules such as integrin $\alpha 4 \beta 1$ and CD62L. By clarifying the function of IL-33 in trophoblast cells, the present study may provide an insight into the rational design of strategy to cure the pregnancy-associated diseases, such as recurrent spontaneous abortion and pregnancy trophoblast diseases.

\section{Discussion}

Pregnancy is a natural physiological process whereby the mother usually does not reject the semi-allogeneic fetus carrying paternal antigens, allowing it to absorb nutrients for growth and development in the uterus, all of which depend on the unique immune tolerance microenvironment around the maternal-fetal interface where nutrient exchange with the mother occurs through the placenta. Subsequent differentiation, development, maturation and delivery of the fetus rely on the accurate molecular regulation of biological functions at the maternal-fetal interface. Immunocompetent cells of the decidua can develop immune tolerance regulated by hormones or cytokines at the maternal-fetal interface $(23,24)$. Immunosuppressed
A
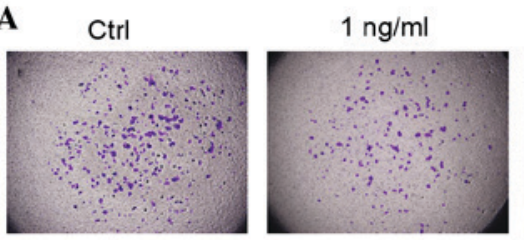

$100 \mathrm{ng} / \mathrm{m}$ rhlL-33

$\mathbf{B}$

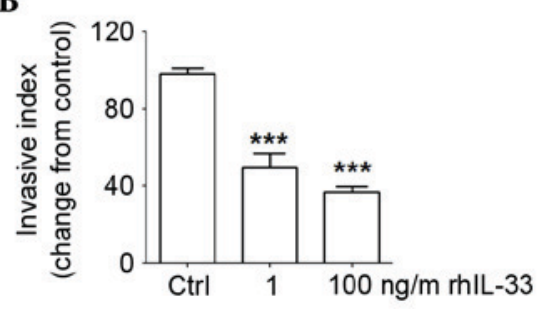

Figure 4. IL-33 inhibits invasiveness of JEG3 cells. (A and B) JEG3 cells were treated with rhIL-33 (1 or $100 \mathrm{ng} / \mathrm{ml})$ for $48 \mathrm{~h}$, with the vehicle as the negative control. The invasion of JEG3 cells was detected by Matrigel invasion assay. Magnification, x100. The data are expressed as the mean \pm standard error. ${ }^{* * *} \mathrm{P}<0.01$ vs. control group. IL-33, interleukin 33; Ctrl, control; rhIL-33, recombinant human IL-33.

states based on maternal T-helper (Th)2 immunity, which is mediated by various cytokines and chemotactic factors, are crucial to the maintenance of pregnancy. Th1/Th2 immunity disorders frequently result in spontaneous abortion in the early phase, and premature birth in the later period, in addition to an increase in the risk of pregnancy complications (25).

IL-33 is an important regulatory factor in Th1/Th2 immunity primarily through binding to its cognate receptor ST2 to induce cells to secrete Th2-type cytokines, such as IL-4 and IL-10. Following induction of the Th2-type immunity, IL-33 contributes to the maternal immune tolerant to the fetus and benefiting pregnancy $(13,26)$. A previous study identified that IL-33 protein was expressed in primary early pregnancy decidual stromal cells in addition to in trophoblast cells (21), which was consistent with the report by Fock et al (22). The current study demonstrated that IL-33 from macrophages could activate AKT and the ERT1/2 signaling pathway, which facilitated the proliferation of trophoblast cells. In the present study, the location of IL-33 and its receptor ST2 were analyzed in first-trimester villi tissues. It was verified that IL-33 and additionally its specific receptor ST2 were expressed in trophoblasts. Therefore, it was hypothesized that IL-33 and ST2 served vital roles in the formation and maintenance of placenta during normal pregnancy.

Following this, the expression of ST2 was detected in four human trophoblast cell lines (JAR, BeWo, JEG3 and HTR8) with FCM analysis, identifying that the BeWo had the highest expression, and HTR8 the lowest. Furthermore, previous studies (27-29) indicated that each trophoblast cell possessed different abilities of adhesion and invasiveness. JEG3 presented the highest adhesion (52\%) and invasion into the bottom stroma. The adhesive rate of BeWo cells was $\sim 37 \%$, and it could penetrate the epithelium, whereas these cells were not able to invade into the stroma. JAR had a lower degree of adhesion (12\%) and exhibited minimal invasion into the stroma (27). According to the adhesion and invasion characteristics of the cells mentioned above, the following investigation in the present study used the JEG3 cell line due to it possessing 

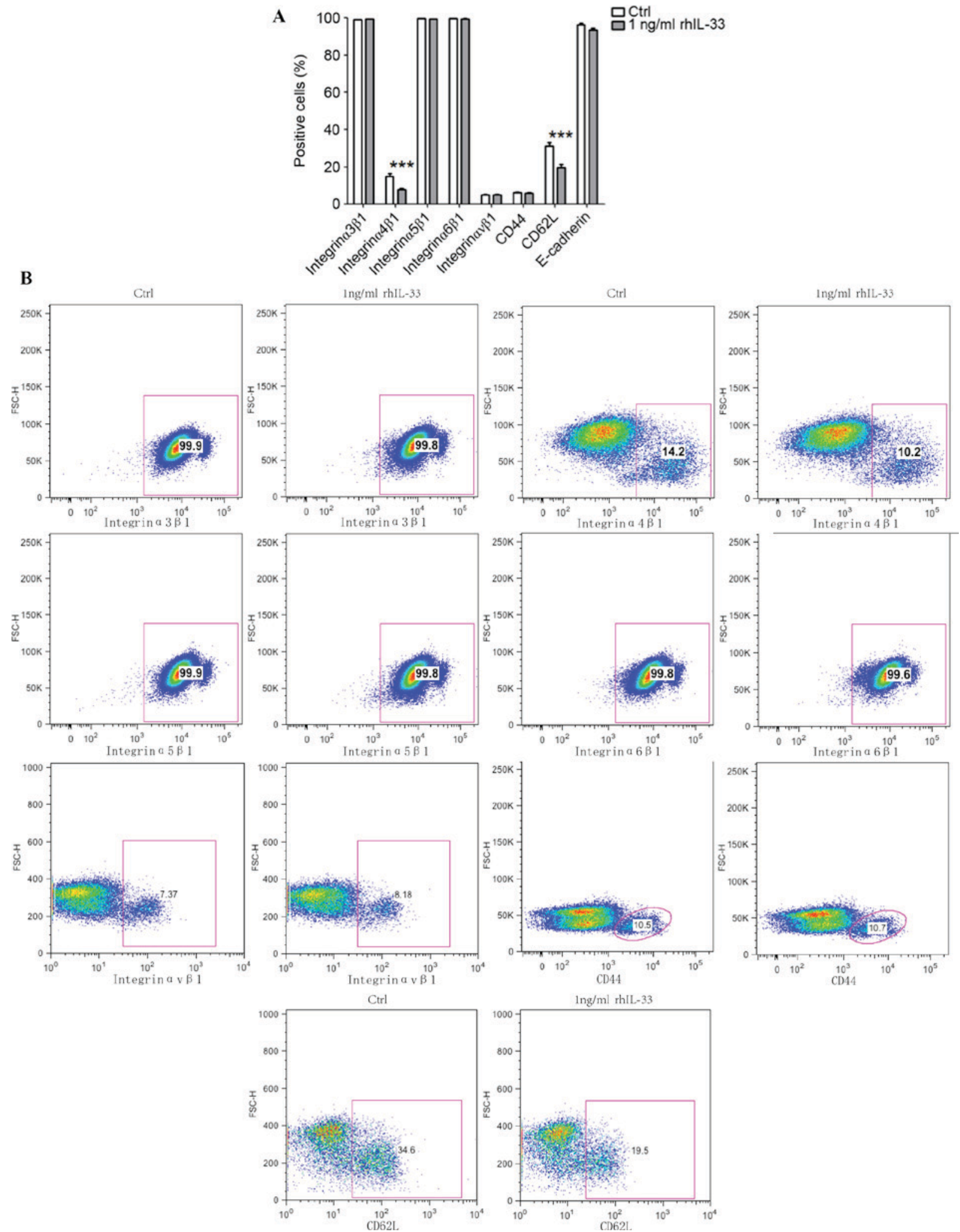

Figure 5. IL-33 decreases the expression of integrin $\alpha 4 \beta 1$ and CD62L on JEG3 cells. Following stimulation with $1 \mathrm{ng} / \mathrm{ml}$ rhIL-33 for $48 \mathrm{~h}$, the adhesion and invasion-related molecules (integrins, E-cadherin, CD62L and CD44) on JEG3 cells were analyzed by flow cytometry, with the vehicle as the negative control (A) Quantification of flow cytometry data. (B) Scatter plots of flow cytometry data. The data are expressed as the mean \pm standard error. ${ }^{* * *} \mathrm{P}<0.01$ vs. control group. IL-33, interleukin 33; rhIL-33, recombinant human IL-33; Ctrl, control.

the strongest adhesion and invasiveness to the uterus, and the fact that it could express ST2.

IL-33 was identified to suppress the adhesion and invasion of JEG3 cells. Following the decidualization of the endometrium, stromal cells produce abundant fibronectin, laminin, collagen IV and heparan sulfate proteoglycans to form a special extracellular stromal microenviroment, which benefits the biological function of trophoblast cells $(30,31)$. The conducted 
adhesion assay demonstrated that, in the presence of exogenous rhIL-33, the adhesion of JEG3 cells to the ECM components, such as fibronectin, laminin I, collagen IV and fibrinogen was significantly repressed. Furthermore, following the addition of exogenous rhIL-33, JEG3 cell invasiveness measured by Transwell assay was reduced. These results indicated that IL-33 suppressed the invasion of trophoblast cells, suggesting that IL-33 might regulate the invasion degree of trophoblast cells into maternal decidua for avoiding excessive cell invasion.

Therefore, the authors suggest that, in the first trimester of pregnancy, the effect of IL-33 is multifaceted. In normal pregnancy, IL-33 expressed at the maternal-fetal interface not only regulates the function of decidual stromal cells, involved in the mechanism of maternal-fetal immune regulation, but also takes part in regulating the proliferation, invasion and adhesion of trophoblasts. IL-33 achieves this through collaborating with other cytokines at the maternal-fetal interface to maintain the balance and limit the excessive invasion of trophoblast cells.

Subsequently, the potential mechanisms might be IL-33 affecting cell adhesion and invasion-related molecules expressed in JEG3 cells. These data suggested that the trophoblast cell line JEG3 highly expressed integrin $\alpha 3 \beta 1$, integrin $\alpha 5 \beta 1$, integrin $\alpha 6 \beta 1$ and E-cadherin, and integrin $\alpha 4 \beta 1$ and CD62L were lowly expressed, while CD44 and integrin $\alpha v \beta 3$ were nearly unexpressed. Following stimulation with exogenous rhIL-33, the expression of $\alpha 4 \beta 1$ and CD62L became notably decreased. A previous study indicated that the expression of trophoblast cell adhesion molecules (CAMs) was predominantly modulated by autocrine and paracrine factors, such as epidermal growth factors, insulin-like growth factors and transforming growth factors. In addition, inflammatory factors, such as TNF $2 \alpha$, IL-21 $\alpha$ and IL-21 $\beta$ may influence the expression of CAMs of trophoblast cells. Cytokines, such as interleukin (IL-1, 3, 4 and 8 ), tumor necrosis factor (TNF- $\alpha, \beta)$, interferon (IFN- $\gamma$ ) and inflammatory mediators (leukotriene, histamine and thrombin), in addition to cell growth, development, differentiation and metabolism state, may regulate the expression of CAMs (32-34). As IL-33 could inhibit cell adhesion and invasiveness, it appears that IL-33 decreases the adhesion and invasion of trophoblast cells possibly through downregulating the expression of integrin $\alpha 4 \beta 1$ and CD62L. However, the mechanism by which IL-33 affects integrin $\alpha 4 \beta 1$ and CD62L remains unclear. Therefore, a functional linkage between IL-33 and CAMs may be involved in invasion by trophoblast cells.

In summary, IL-33 is a pro-inflammatory cytokine, involved in immune and inflammatory reaction, in addition to regulating biological functions of many cell types. According to the present study, IL-33 may regulate the adhesion and invasion of trophoblast cells by regulating the expression of the surface adhesion molecules. Regulation of these factors maintain the embryo implantation, placental formation and placenta function during pregnancy, and also accompany additional molecules to control the extent of invasion, in order to guarantee the normal function of the placenta, and act against pregnancy-associated diseases.

\section{Acknowledgements}

The present study was supported by the National Natural Science Foundation of China (NSFC; grant no. 81671460),
National 863 Program (grant no. 2014AA020706), the Shanghai Outstanding Academic Leaders Program (grant no. 15XD1500900); Program for Shanghai leaders; Training Program for excellent academic leaders of Shanghai Health System (grant no. XBR2013093); the Innovation Projects of Shanghai Municipal Education Commission (grant no. 14ZZ011); the Foundation of Shanghai Science and Technology Committee (grant no. 134119a4300; to Professor Li-Ping Jin); NSFC (grant no. 81300552; to Mrs. Deng-Xuan Fan); and the Foundation of Shanghai First Maternity and Infant Hospital, Tongji University School of Medicine (grant no. 2016A17; to Mr. Xiao-Hui Wang).

\section{References}

1. Carson DD, Bagchi I, Dey SK, Enders AC, Fazleabas AT, Lessey BA and Yoshinaga K: Embryo implantation. Dev Biol 223: 217-237, 2000

2. Bowen JM, Chamley L, Mitchell MD and Keelan JA: Cytokines of the placenta and extra-placental membranes: Biosynthesis, secretion and roles in establishment of pregnancy in women. Placenta 23: 239-256, 2002.

3. Knöfler M: Critical growth factors and signalling pathways controlling human trophoblast invasion. Int J Dev Biol 54: 269-280, 2010.

4. Knöfler M and Pollheimer J: IFPA award in placentology lecture: Molecular regulation of human trophoblast invasion. Placenta 33 (Suppl): S55-S62, 2012.

5. Tessier DR, Yockell-Lèlievre J and Gruslin A: Uterine spiral artery remodeling: The role of uterine natural killer cells and extravillous trophoblasts in normal and high-risk human pregnancies. Am J Reprod Immunol 74: 1-11, 2015.

6. Chen JZ, Sheehan PM, Brennecke SP and Keogh RJ: Vessel remodelling, pregnancy hormones and extravillous trophoblast function. Mol Cell Endocrinol 349: 138-144, 2012.

7. Al-Khan A, Bulmer JN, Chantraine F, Chen CP, Chen Q, Collins S, Cotechini T, Fitzgerald JS, He M, Holland O, et al: IFPA meeting 2012 workshop report III: Trophoblast deportation, gestational trophoblastic disease, placental insufficiency and fetal growth restriction, trophoblast over-invasion and accreta-related pathologies, placental thrombosis and fibrinolysis. Placenta 34 (Suppl): S11-S16, 2013.

8. Lash GE: Molecular cross-talk at the feto-maternal interface. Cold Spring Harb Perspect Med 5: pii: a023010, 2015.

9. Highet AR, Khoda SM, Buckberry S, Leemaqz S, Bianco-Miotto T, Harrington E, Ricciardelli C and Roberts CT: Hypoxia induced HIF-1/HIF-2 activity alters trophoblast transcriptional regulation and promotes invasion. Eur J Cell Biol 94: 589-602, 2015

10. Bazer FW, Wu G, Johnson GA and Wang X: Environmental factors affecting pregnancy: Endocrine disrupters, nutrients and metabolic pathways. Mol Cell Endocrinol 398: 53-68, 2014.

11. Wang S, Ding L, Liu SS, Wang C, Leng RX, Chen GM, Fan YG, Pan HF and Ye DQ: IL-33: A potential therapeutic target in autoimmune diseases. J Investig Med 60: 1151-1156, 2012.

12. Beklen A and Tsaous Memet G: Interleukin-1 superfamily member, interleukin-33, in periodontal diseases. Biotech Histochem 89: 209-214, 2014.

13. Schmitz J, Owyang A, Oldham E, Song Y, Murphy E, McClanahan TK, Zurawski G, Moshrefi M, Qin J, Li X, et al: IL-33, an interleukin-1-like cytokine that signals via the IL-1 receptor-related protein ST2 and induces T helper type 2associated cytokines. Immunity 23: 479-490, 2005.

14. Miller AM: Role of IL-33 in inflammation and disease. J Inflamm (Lond) 8: 22, 2011.

15. Jovanovic IP, Pejnovic NN, Radosavljevic GD, Pantic JM, Milovanovic MZ, Arsenijevic NN and Lukic ML: Interleukin-33/ST2 axis promotes breast cancer growth and metastases by facilitating intratumoral accumulation of immunosuppressive and innate lymphoid cells. Int J Cancer 134: 1669-1682, 2014.

16. Chen SF, Nieh S, Jao SW, Wu MZ, Liu CL, Chang YC and Lin YS: The paracrine effect of cancer-associated fibroblast-induced interleukin-33 regulates the invasiveness of head and neck squamous cell carcinoma. J Pathol 231: 180-189, 2013. 
17. Chow JY, Wong CK, Cheung PF and Lam CW: Intracellular signaling mechanisms regulating the activation of human eosinophils by the novel Th2 cytokine IL-33: Implications for allergic inflammation. Cell Mol Immunol 7: 26-34, 2010.

18. Demyanets S, Konya V, Kastl SP, Kaun C, Rauscher S, Niessner A Pentz R, Pfaffenberger S, Rychli K, Lemberger CE, et al: Interleukin-33 induces expression of adhesion molecules and inflammatory activation in human endothelial cells and in human atherosclerotic plaques. Arterioscler Thromb Vasc Biol 31: 2080-2089, 2011.

19. Suzukawa M, Koketsu R, Iikura M, Nakae S, Matsumoto K, Nagase H, Saito H, Matsushima K, Ohta K, Yamamoto K and Yamaguchi M: Interleukin-33 enhances adhesion, CD11b expression and survival in human eosinophils. Lab Invest 88: 1245-1253, 2008.

20. Iikura M, Suto H, Kajiwara N, Oboki K, Ohno T, Okayama Y, Saito H, Galli SJ and Nakae S: IL-33 can promote survival, adhesion and cytokine production in human mast cells. Lab Invest 87: 971-978, 2007.

21. Hu WT, Li MQ, Liu W, Jin LP, Li DJ and Zhu XY: IL-33 enhances proliferation and invasiveness of decidual stromal cells by up-regulation of CCL2/CCR 2 via NF- $x \mathrm{~B}$ and ERK1/2 signaling. Mol Hum Reprod 20: 358-372, 2014

22. Fock V, Mairhofer M, Otti GR, Hiden U, Spittler A, Zeisler H, Fiala C, Knöfler M and Pollheimer J: Macrophage-derived IL-33 is a critical factor for placental growth. J Immunol 191: 3734-3743, 2013

23. Du MR, Wang SC and Li DJ: The integrative roles of chemokines at the maternal-fetal interface in early pregnancy. Cell Mol Immunol 11: 438-448, 2014.

24. Arck PC and Hecher K: Fetomaternal immune cross-talk and its consequences for maternal and offspring's health. Nat Med 19: $548-556,2013$.
25. Piccinni MP: T cell tolerance towards the fetal allograft. J Reprod Immunol 85: 71-75, 2010

26. Salker MS, Nautiyal J, Steel JH, Webster Z, Sućurović S, Nicou M, Singh Y, Lucas ES, Murakami K, Chan YW, et al: Disordered IL-33/ST2 activation in decidualizing stromal cells prolongs uterine receptivity in women with recurrent pregnancy loss. PLoS One 7: e52252, 2012.

27. Grümmer R, Hohn HP, Mareel MM and Denker HW: Adhesion and invasion of three human choriocarcinoma cell lines into human endometrium in a three-dimensional organ culture system. Placenta 15: 411-429, 1994.

28. Hohn HP, Linke M and Denker HW: Adhesion of trophoblast to uterine epithelium as related to the state of trophoblast differentiation: In vitro studies using cell lines. Mol Reprod Dev 57: $135-145,2000$.

29. Wu LZ, Liu XL and Xie QZ: Osteopontin facilitates invasion in human trophoblastic cells via promoting matrix metalloproteinase-9 in vitro. Int J Clin Exp Pathol 8: 14121-14130, 2015.

30. Oefner CM, Sharkey A, Gardner L, Critchley H, Oyen M and Moffett A: Collagen type IV at the fetal-maternal interface. Placenta 36: 59-68, 2015.

31. Smith SD, Choudhury RH, Matos P, Horn JA, Lye SJ, Dunk CE, Aplin JD, Jones RL and Harris LK: Changes in vascular extracellular matrix composition during decidual spiral arteriole remodeling in early human pregnancy. Histol Histopathol 31: 557-571, 2015.

32. Aplin JD, Jones CJ and Harris LK: Adhesion molecules in human trophoblast-a review. I. Villous trophoblast. Placenta 30: 293-298, 2009

33. Aplin JD: Adhesion molecules in implantation. Rev Reprod 2: 84-93, 1997.

34. Harris LK, Jones CJ and Aplin JD: Adhesion molecules in human trophoblast-a review. II. Extravillous trophoblast. Placenta 30: 299-304, 2009. 